\title{
Accretion-Ejection Instability in magnetized disks: Feeding the corona with Alfvén waves
}

\author{
P. Varnière and M. Tagger \\ DSM/DAPNIA/Service d'Astrophysique (CNRS URA 2052), CEA Saclay, 91191 Gif-sur-Yvette, France \\ Received 9 January 2002 / Accepted 30 July 2002

\begin{abstract}
We present a detailed calculation of the mechanism by which the Accretion-Ejection Instability can extract accretion energy and angular momentum from a magnetized disk, and redirect them to its corona. In a disk threaded by a poloidal magnetic field of the order of equipartition with the gas pressure, the instability is composed of a spiral wave (analogous to galactic ones) and a Rossby vortex. The mechanism detailed here describes how the vortex, twisting the footpoints of field lines threading the disk, generates Alfvén waves propagating to the corona. We find that this is a very efficient mechanism, providing to the corona (where it could feed a jet or a wind) a substantial fraction of the accretion energy.
\end{abstract}

Key words. accretion, accretion disks - instabilities - MHD - waves - galaxies: jets

\section{Introduction}

MHD models have shown that jets can be very efficient to carry away the accretion energy and angular momentum extracted by turbulence from accretion disks (Blandford \& Payne 1982; Lovelace et al. 1987; Pelletier \& Pudritz 1992), if the disk is threaded by a poloidal magnetic field. This fits with the observation that accretion and ejection are intimately connected in objects ranging from protostellar disks to X-ray binaries and AGNs. However these models, based on self-similar analytical computations or on numerical simulations, most often start at the upper surface of the disk. Although more recent works (Ferreira \& Pelletier 1993a, 1993b, 1995; Casse \& Ferreira 2000) find solutions connecting continuously the disk and the jet, these solutions are heavily constrained by conflicting requirements. These can be traced, in part, to the fact that disk models, whether they rely on specific instability mechanisms or on the assumption of a turbulent viscosity, imply that the accretion energy and angular momentum are transported radially outward. They must thus somehow be redirected upward to feed the jet.

The Accretion-Ejection Instability (AEI) of magnetized accretion disks, presented by Tagger and Pellat (1999, hereafter TP99), could provide a solution to this difficulty. It occurs in the inner region of the disk, in the configuration assumed by the MHD models of jets (i.e. a disk threaded by a poloidal field of the order of equipartition with the gas thermal pressure), and it has the unique property that energy and momentum extracted from the disk can be emitted vertically as Alfvén waves propagating along magnetic field lines to the corona of the disk.

Send offprint requests to: $\mathrm{P}$. Varnière,

e-mail: pvarni@discovery.saclay.cea.fr
Thus they could provide a source for a jet or a wind formed in the corona.

This ability to emit the energy and angular momentum as Alfven wave was recognized in TP99, and indeed justified the name given to the instability. However this possibility was shown only in a WKB approximation, valid away from the region (the corotation radius, where the wave rotates at the same velocity as the gas) where Alfvén wave emission is most efficient. The WKB result was found divergent at corotation, providing a good indication that this mechanism of vertical emission could be quite efficient.

The goal of this paper is to present a more general derivation, valid in the corotation region. We use a description of the waves in three dimensions (whereas TP99 was basically a model averaged over the disk thickness). We are thus able to give an explicit computation of the Alfvén wave emission mechanism and of its efficiency. The main unknown to be solved for at this stage is the fraction of the accretion energy and angular momentum, extracted from the disk by the instability, which will end up emitted to the corona.

The result we present is quite limited: the constraints of giving an analytical derivation force us to use a very simplified magnetic field geometry, namely an initially constant and vertical field, and a more realistic one would certainly affect the result. Appendix A is dedicated to the case of a radially varying $B_{z}$ field. We show that the present computation may be apply in the case of a slowly variable $B$ field. On the other hand, the result we obtain is interesting in itself: we will show that, in linear theory, the flux of the Alfvén waves is again divergent at corotation. Although we discuss how it can be regularized, our interpretation will be that the efficiency of the mechanism is indeed quite high, but that we are reaching the limits of linear 


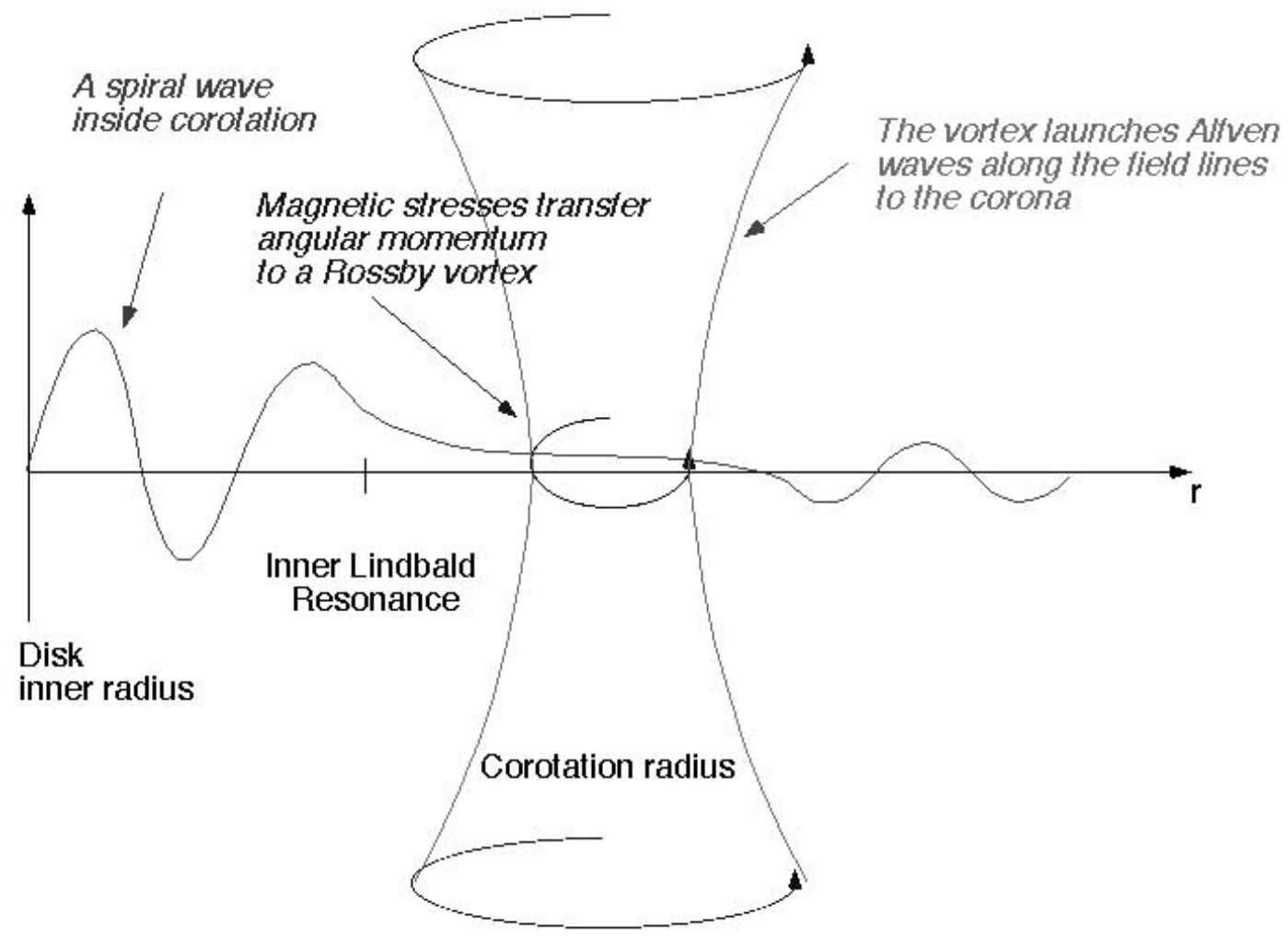

Fig. 1. The structure of the instability is shown here schematically as a function of radius. It is formed of a standing spiral density wave in the inner part of the disk, coupled to a Rossby vortex it excites at its corotation radius. The spiral grows by extracting energy and angular momentum from the disk, and depositing them in the Rossby vortex; the latter in turn generates Alfvén waves propagating toward the corona of the disk.

theory and that the true result will most certainly be determined by self-consistent non-linear effects. The present work should thus be viewed as an exploration of the complex physics involved and of its potential efficiency, which will then have to be treated by non-linear simulations. The paper is structured as follows: in the next section we will briefly review the main properties of the instability, and its interest to explain the lowfrequency Quasi-Periodic Oscillation (QPO) of X-ray binaries. Section 3 will present the system of equations to be solved, and Sect. 4 their combination into a variational form containing the physics of the problem. In Sect. 5 we will compute the Alfvén wave flux, and we will discuss the significance of this result in Sect. 6. In Appendix A we will present the variational form we obtain in a more general geometry with a radially varying $B_{z}$ and the limitation it implies.

\section{The Accretion-Ejection Instability}

We will give here a short review of the main characteristics of the AEI, and refer the interested reader to TP99, or to Varnière et al. (2002) and Rodriguez et al. (2002) where a detailed comparison with the low-frequency QPO of black-hole binaries is given. Non-linear MHD simulations were performed by Caunt \& Tagger (2001). The AEI is essentially a spiral instability, similar to galactic ones but driven by magnetic stresses rather than self-gravity. It affects the inner region of an accretion disk threaded by a poloidal magnetic field of the order of equipartition with the thermal pressure of the gas $\left(\beta=8 \pi p / B^{2}\right.$ of the order of unity), i.e. the configuration and magnitude used in most MHD jet models.

The instability is composed of a spiral density wave and a Rossby wave it generates at its corotation radius (the radius where the wave rotates at the same velocity as the gas). The spiral forms a standing pattern between the inner edge of the disk and the corotation radius. Because of differential rotation, it couples to the Rossby wave, i.e. the spiral and Rossby waves exchange energy and angular momentum in the corotation region where, because of differential rotation they loose their separate identities.

In this process the Rossby wave also forms a standing vortex, and the spiral grows by storing in it the energy and angular momentum it extracts from the inner region of the disk (thus causing accretion).

In a thin disk in vacuum, the process stops there. This is the case in galaxies and recently Fridman et al. (2001) observe and analyse such a vortex in NGC 157 . However one must remember that the Rossby vortex represents a torsion of the footpoints of the field lines threading the disk. Thus if the disk is covered by a low-density corona, this torsion will propagate vertically along the field lines as Alfvén waves: these will thus in turn transport a fraction of the accretion energy and angular momentum to the corona, where they could provide a source for a jet or an outflow. The whole process is shown schematically in Fig. 1. 


\section{Linearized equations}

\subsection{Unperturbed equilibrium}

As in previous works, we consider the very simple setup of an infinitely thin disk threaded by a vertical magnetic field adding this time the hypothesis that it is radially constant. Here however the disk is embedded in a low density corona. This simplified geometry will be enough to fully characterize the emission of Alfvén waves in the corona. The equilibrium field, $\boldsymbol{B}^{\mathbf{0}}=B_{0} \boldsymbol{e}_{z}$, is assumed to be of the order of equipartition with the gas pressure (plasma $\beta=8 \pi p / B_{0}^{2} \sim 1$ ) in the disk. The case of a radially varying $B_{0}$ will be studied in Appendix A.

We choose to present here results with a constant $B^{0}$ because assuming that $B^{0}$ depends on $r$ creates at equilibrium a radial magnetic pressure force which can easily become dominant in the corona, since the other forces (gravity, Coriolis, pressure) act on a very low density. As a result a realistic equilibrium will in general include a flow along the field lines, and lead to a complex configuration limiting our ability to extract analytical results unless artificial assumptions are made.

On the other hand instability requires, as found in TP99:

$\frac{\partial}{\partial s}\left(\frac{W \Sigma}{B_{0}^{2}}\right)>0$

where $W=\kappa^{2} / 2 \Omega$ and $\kappa=\Omega \sim r^{-3 / 2}$ in a Keplerian disk. Thus, for this quantity to grow with $r$, our equilibrium model needs to have a disk surface density growing with $r$, fast enough for this condition to be fulfilled. This appears as an ad hoc model which does not claim to represent a real disk. We emphasize however that it is physically consistent, so that our computation of the Alfvén wave flux remains exact and will allow us to explore physics which applies to more general disk models ${ }^{1}$.

\subsection{Pertubations}

We work in cylindrical coordinates $[(s=\ln r), \theta, z]$. We consider linear perturbations, described by Lagrangian displacements $\xi$ with:

$\boldsymbol{V}=\frac{\mathrm{d}}{\mathrm{d} t} \boldsymbol{\xi}+(\boldsymbol{\xi} \cdot \boldsymbol{\nabla}) \boldsymbol{V}_{0}$

where $\boldsymbol{V}$ is the perturbed velocity, $\boldsymbol{V}_{0}=r \Omega(r)$ is the equilibrium rotation velocity, and

$\frac{\mathrm{d}}{\mathrm{d} t}=\frac{\partial}{\partial t}+\boldsymbol{V}_{0} \cdot \boldsymbol{\nabla}$

In TP99 the instability was studied by giving a full solution for its spatial structure in cylindrical geometry; the emission of Alfvén waves was then computed perturbatively, far from the corotation radius, in the WKB limit. The waves were described by their compressional and torsional components,

$D=\nabla_{\perp} \cdot V_{\perp} \quad$ and $\quad R=\nabla_{\perp} \times V_{\perp}$

${ }^{1}$ It is worth noticing (J. Ferreira, private communication) that the self-similar assumption used in MHD models of jets, following Blandford and Payne, always results in this derivative being positive and equal to $+1 / 2$, as a result of the self-similarity laws. Thus all these models are unstable to the AEI. where the subscript $\perp$ describes the components of a vector in the plane of the disk. We find this description inconvenient here and rather write the displacement as:

$B_{0}=-\nabla_{\perp} \Phi-e_{z} \times \nabla_{\perp} \Psi$

where again $\Phi$ and $\Psi$ represent the compressional and torsional parts of the displacement.

From this we write the induction equation,

$\partial_{\mathrm{t}} \boldsymbol{B}+\boldsymbol{\nabla} \times(\boldsymbol{V} \times \boldsymbol{B})=0:$

giving the perturbed magnetic field as:

$\boldsymbol{B}_{\perp}^{1}=\left(\boldsymbol{B}^{0} \cdot \nabla\right) \boldsymbol{\xi}_{\perp}=B_{0} \partial_{z} \boldsymbol{\xi}_{\perp}$

and from $\boldsymbol{\nabla} \cdot B_{1}=0$ :

$B_{z}^{1}=-\nabla_{\perp} \cdot\left(B_{0} \xi_{\perp}\right)$.

Comparing with the solution of the continuity equation, which gives the perturbed density $\rho=-\boldsymbol{\nabla}_{\perp} \cdot\left(\rho_{0} \boldsymbol{\xi}_{\perp}\right)$, one notes that Eq. (2) is an equation of conservation for the vertical magnetic flux.

We consider perturbations varying as $\exp [i(m \theta-\omega t)]$ (so that $m$ will be the number of arms of the spiral). We define a de-dimensionalized perpendicular gradient,

$\tilde{\boldsymbol{\nabla}}_{\perp} X=r \boldsymbol{\nabla}_{\perp} X=\partial_{\mathrm{s}} X \boldsymbol{e}_{\mathrm{r}}+i m X \boldsymbol{e}_{\vartheta}$.

We finally get the perturbed magnetic field:

$\boldsymbol{B}^{1}=\frac{1}{r}\left(\begin{array}{c}-\partial_{z, s} \Phi+i m \partial_{z} \Psi \\ -i m \partial_{z} \Phi-\partial_{z, s} \Psi \\ \frac{1}{r} \tilde{\nabla}_{\perp}^{2} \Phi\end{array}\right)$.

As in TP99, our goal is to derive from the MHD equations a variational form. Its real (self-adjoint) part represents the energy of the perturbations and describes their structure in the disk. Amplification and damping appear through imaginary terms, representing a flux of energy: either at the boundaries of the system (as outgoing waves) or at the corotation resonance. The classical physics of spiral waves in disks with differential rotation is that they have positive energy beyond the corotation resonance $\left(r_{\mathrm{C}}\right.$ such that $\left.m \Omega\left(r_{\mathrm{C}}\right)=\omega\right)$, and negative energy inside it: at $r<r_{\mathrm{C}}$ the gas rotates faster than the wave, whose presence decreases the total energy of the system (it releases more gravitational energy than it costs kinetic energy).

The spiral can thus be amplified if positive energy is emitted beyond corotation (as a spiral wave emitted outward: this is the Swing mechanism, responsible for the amplification of galactic spirals), or stored in a Rossby vortex at the corotation radius: in TP99 this was shown to result, in a magnetized disk, in the Accretion-Ejection Instability. This corotation resonance introduces, in the variational form of TP99, a pole (a denominator proportional to $[\omega-m \Omega(r)]$, singular at corotation). This pole contributes, following the Landau prescription familiar in plasma physics, an imaginary term which represents the energy exchanged with the Rossby vortex.

In TP99 it was also found that, when one takes into account a low-density corona above the disk, Alfvén waves are emitted toward the corona; their contribution was computed in 
a WKB approximation, valid only away from corotation. This had of course a limited interest, since we expect the Alfvén waves to be strongest at corotation where the torsional motion associated with the vortex is strongest. Here we will present a more general formulation, showing how the energy flux of the Alfvén waves appears as an additional imaginary term in the variational form, generated at the vertical boundaries of the disk. This will allow us to compute explicitly the Alfvén wave flux at corotation.

In terms of the Lagrangian displacement, the linearized Euler equation for a perturbation varying as $\mathrm{e}^{i(m \vartheta-\omega t)}$ can be written as:

$$
\begin{aligned}
& \left(-\tilde{\omega}^{2}+2 \Omega \Omega^{\prime}\right) \xi_{r}+2 i \tilde{\omega} \Omega \xi_{\vartheta}=f_{r} \\
& -\tilde{\omega}^{2} \xi_{\vartheta}-2 i \tilde{\omega} \Omega \xi_{r}=f_{\vartheta} \\
& -\tilde{\omega}^{2} \xi_{z}=f_{z}
\end{aligned}
$$

where $\boldsymbol{f}=\boldsymbol{F} / \rho_{0}, \boldsymbol{F}$ is the force acting on the system, $\rho_{0}(s, z)$ is the equilibrium density, $\tilde{\omega}(s)=\omega-m \Omega(s)$, and the prime denotes differentiation with respect to $s$. In terms of $\Phi$ and $\Psi$ the $r$ and $\vartheta$ components of these equations become:

$$
\begin{aligned}
\left(\tilde{\omega}^{2}-2 \Omega \Omega^{\prime}\right) \partial_{s} \Phi+2 m \tilde{\omega} \Omega \Phi & \\
& -2 i \tilde{\omega} \Omega \partial_{s} \Psi-i m\left(\tilde{\omega}^{2}-2 \Omega \Omega^{\prime}\right) \Psi=r B_{0} f_{r}
\end{aligned}
$$

$$
2 i \tilde{\omega} \Omega \partial_{s} \Phi+i m \tilde{\omega}^{2} \Phi+\tilde{\omega}^{2} \partial_{s} \Psi+2 m \tilde{\omega} \Omega \Psi=r B_{0} f_{\vartheta}
$$

For simplicity we will neglect the pressure stress, since it was found in TP99 to be negligible, compared to the magnetic ones, in the physics of the instability. It could be added without change on the emission of Alfvén waves. On the other hand, this means that we do not consider here the slow magnetosonic wave which could also propagate upward from the disk. We have two reasons for that: the first one is that this would lead to a very complex problem, which goes beyond the goals of the present paper; it would force us to consider the detailed physics at the slow magnetosonic point above the disk surface (see e.g. Ferreira \& Pelletier 1995, and references therein), where the gas is first extracted from the disk. This means that, in stationary MHD models of jets, the physics of the slow magnetosonic wave concerns the mass loading of the field lines, rather than the acceleration of the jet which is more associated with the Alfvén wave. Our second reason is that we will show below how the Alfvén wave is mainly excited in a resonant layer at the corotation radius, by the singular Rossby vortex generated in the disk. No such singularity concerns the slow wave, which should thus not be so important for us. We thus postpone its consideration to future work. In the same manner, consistent with the thin disk approximation, we will neglect the vertical component of the Euler equation.

Linearizing the contribution of magnetic stresses, $(\boldsymbol{j} \times \boldsymbol{B}) / \rho$, we find:

$f=\frac{\boldsymbol{j}^{1} \times \boldsymbol{B}^{0}}{\rho_{0}}$ where the subscripts 0 and 1 note equilibrium and perturbed values. We thus get the expression of the magnetic stresses:

$$
\begin{aligned}
& B_{0} f_{r}=V_{\mathrm{A}}^{2}\left(\partial_{z} B_{r}^{1}-\partial_{r} B_{z}^{1}\right) \\
& B_{0} f_{\vartheta}=V_{\mathrm{A}}^{2}\left(\partial_{z} B_{\vartheta}^{1}-\partial_{\vartheta} B_{z}^{1}\right)
\end{aligned}
$$

(where $\left.V_{\mathrm{A}}^{2}(z)=B_{0}^{2} / 4 \pi \rho_{0}\right)$; we express this in terms of $\Phi$ and $\Psi$, and get from Eqs. (4), (5):

$$
\begin{aligned}
r B_{0}\left[\left(-\tilde{\omega}^{2}+2 \Omega \Omega^{\prime}\right) \xi_{r}\right. & \left.+2 i \tilde{\omega} \Omega \xi_{\vartheta}\right]= \\
& -V_{\mathrm{A}}^{2}\left(\partial_{s} \nabla^{2} \Phi-i m \partial_{z^{2}} \Psi\right) \\
r B_{0}\left[-\tilde{\omega}^{2} \xi_{\vartheta}-2 i \tilde{\omega} \Omega \xi_{r}\right] & =-V_{\mathrm{A}}^{2}\left(\partial_{s, z^{2}} \Psi+i m \nabla^{2} \Phi\right) .
\end{aligned}
$$

\section{The system}

In order to obtain the new variational form, we first write a quadratic form built from the divergence and the curl of $\lambda B_{0}$ times the Euler equation, leaving $\lambda$ unspecified for the moment.

We first apply the operator $r^{2} \tilde{\nabla}_{\perp} \cdot \lambda B_{0}$ to the Euler equations, i.e. we compute:

$\frac{\partial}{\partial s}\left(r \lambda B_{0}[10]\right)+i m r \lambda B_{0}[11]$

we get:

$$
\begin{aligned}
& \tilde{\nabla}_{\perp}\left(\lambda \tilde{\omega}^{2} \tilde{\nabla}_{\perp} \Phi\right)-2 \partial_{s}\left(\lambda \Omega \Omega^{\prime} \partial_{s} \Phi\right) \\
& +2 m \partial_{s}(\lambda \Omega \tilde{\omega}) \Phi-2 i \tilde{\nabla}_{\perp}\left(\lambda \tilde{\omega} \Omega \tilde{\nabla}_{\perp} \Psi\right) \\
& -i m \partial_{s}\left(\lambda \tilde{\omega}^{2}\right) \Psi+2 i m \partial_{s}\left(\lambda \Omega \Omega^{\prime} \Psi\right) \\
& =-\lambda V_{\mathrm{A}}^{2} \tilde{\nabla}_{\perp}^{2} \nabla^{2} \Phi \\
& \quad-\left(\lambda \partial_{s} V_{\mathrm{A}}^{2}+V_{\mathrm{A}}^{2} \partial_{s} \lambda\right)\left(i m \partial_{z^{2}} \Psi-\partial_{s} \nabla^{2} \Phi\right) .
\end{aligned}
$$

Then applying the operator $r^{2} \tilde{\nabla}_{\perp} \times \lambda B_{0}$ to the Euler equations:

$$
\begin{aligned}
& \tilde{\nabla}_{\perp}\left(\lambda \tilde{\omega}^{2} \tilde{\nabla}_{\perp} \Psi\right)+2 m \tilde{\omega} \partial_{s}(\lambda \Omega) \Psi \\
& +2 i \tilde{\nabla}_{\perp}\left(\lambda \tilde{\omega} \Omega \tilde{\nabla}_{\perp} \Phi\right)+i m \partial_{s}\left(\lambda \tilde{\omega}^{2}\right) \Phi \\
& +2 i m \lambda \Omega \Omega^{\prime} \partial_{s} \Phi \\
& =-\lambda V_{\mathrm{A}}^{2} \partial_{z^{2}} \tilde{\nabla}_{\perp}^{2} \Psi \\
& \quad-\left(\lambda \partial_{s} V_{\mathrm{A}}^{2}+V_{\mathrm{A}}^{2} \partial_{s} \lambda\right)\left(i m \partial_{z^{2}} \Psi-\partial_{s} \nabla^{2} \Phi\right) .
\end{aligned}
$$

In order to get a variational form from these equations we need to get rid of the term in $\left(i m \partial_{z^{2}} \Psi-\partial_{s} \nabla^{2} \Phi\right)$, present in both. This will be the case if $\lambda \partial_{s} V_{\mathrm{A}}^{2}+V_{\mathrm{A}}^{2} \partial_{s} \lambda=0$. We thus make the obvious choice $\lambda=V_{\mathrm{A}}^{-2}(s, z)$, and Eqs. (12), (13) then become:

$\tilde{\nabla}_{\perp}\left(\frac{\tilde{\omega}^{2}}{V_{\mathrm{A}}^{2}} \tilde{\nabla}_{\perp} \Phi\right)-2 \partial_{s}\left(\frac{\Omega \Omega^{\prime}}{V_{\mathrm{A}}^{2}} \partial_{s} \Phi\right)$

$+2 m \partial_{s}\left(\frac{\Omega \tilde{\omega}}{V_{\mathrm{A}}^{2}}\right) \Phi-2 i \tilde{\nabla}_{\perp}\left(\frac{\Omega \tilde{\omega}}{V_{\mathrm{A}}^{2}} \tilde{\nabla}_{\perp} \Psi\right)$

$-i m \partial_{s}\left(\frac{\tilde{\omega}^{2}}{V_{\mathrm{A}}^{2}}\right) \Psi+2 i m \partial_{s}\left(\frac{\Omega \Omega^{\prime}}{V_{\mathrm{A}}^{2}} \Psi\right)$

$=-\tilde{\nabla}_{\perp}^{2} \nabla^{2} \Phi$ 
and:

$\tilde{\nabla}_{\perp}\left(\frac{\tilde{\omega}^{2}}{V_{\mathrm{A}}^{2}} \tilde{\nabla}_{\perp} \Psi\right)+2 m \tilde{\omega} \partial_{s}\left(\frac{\Omega}{V_{\mathrm{A}}^{2}}\right) \Psi$

$+2 i \tilde{\nabla}_{\perp}\left(\frac{\tilde{\omega} \Omega}{V_{\mathrm{A}}^{2}} \tilde{\nabla}_{\perp} \Phi\right)+i m \partial_{s}\left(\frac{\tilde{\omega}^{2}}{V_{\mathrm{A}}^{2}}\right) \Phi$

$+2 i m \frac{\Omega \Omega^{\prime}}{V_{\mathrm{A}}^{2}} \partial_{s} \Phi$

$=-\partial_{z^{2}} \tilde{\nabla}_{\perp}^{2} \Psi$.

We will later put these equations in a more compact form, but use for the moment the present one which is best adapted to a variational formulation.

\subsection{The variational form}

In order to obtain the variational form we will first integrate these equations vertically. Assuming that the disk is covered by a low-density corona, we integrate between $z=-z_{\text {maz }}$ and $+z_{\max }$, chosen well into the corona so that at that height only the Alfvén waves propagate and their vertical propagation can be described in the WKB approximation ${ }^{2}$. We write the integral:

$F \equiv \int_{s_{\min }}^{s_{\max }} \int_{z_{\min }}^{z_{\max }} \Phi^{\star}[14]+\Psi[15]^{\star} \mathrm{d} z \mathrm{~d} s$

$=0$

where $[15]^{\star}$ is the complex conjugate of Eq. (15), and the radial boundaries $s_{\min }$ and $s_{\max }$ will be discussed later. We will find that, thanks to our choice of $\lambda$, this form has the properties we wished: it is composed of a main part, which is variational, and additional terms which can be treated perturbatively to give amplification or damping of the waves.

After some algebra, integrating by parts and grouping terms we get:

$$
\begin{aligned}
& \int_{s_{\min }}^{s_{\max }} \int_{z_{\min }}^{z_{\max }}\left\{\frac{\tilde{\omega}^{2}}{V_{\mathrm{A}}^{2}}\left(\left|\tilde{\nabla}_{\perp} \Psi\right|^{2}-\left|\tilde{\nabla}_{\perp} \Phi\right|^{2}\right)\right. \\
&+2 \frac{\Omega \Omega^{\prime}}{V_{\mathrm{A}}^{2}}\left|\partial_{s} \Phi\right|^{2} \\
&+2 m \partial_{s}\left(\frac{\tilde{\omega} \Omega}{V_{\mathrm{A}}^{2}}\right)|\Phi|^{2} \\
&-2 m \tilde{\omega} \partial_{s}\left(\frac{\Omega}{V_{\mathrm{A}}^{2}}\right)|\Psi|^{2} \\
&=-\left[\int _ { z _ { \operatorname { m i n } } } ^ { z _ { \operatorname { m a x } } } \frac { \mathrm { d } z } { V _ { \mathrm { A } } ^ { 2 } } \left(\tilde{\omega}^{2} \Phi^{\star} \tilde{\nabla}_{\perp} \Phi-2 \Omega \Omega^{\prime} \Phi^{\star} \partial_{s} \Phi\right.\right. \\
&-\tilde{\omega}^{2} \Psi \tilde{\nabla}_{\perp} \Psi \Psi^{\star}+2 i m \Omega \Omega^{\prime} \Phi^{\star} \Psi \\
&\left.\left.-2 i \tilde{\omega} \Omega\left(\Psi \tilde{\nabla}_{\perp} \Phi^{\star}+\Phi^{\star} \tilde{\nabla}_{\perp} \Psi\right)\right)\right]_{s_{\min }}^{s_{\max }} \\
&+\int_{s_{\min }}^{s_{\max }}\left[\Psi^{\star} \partial_{z} \tilde{\nabla}_{\perp}^{2} \Psi\right]_{-z_{\max }}^{z_{\max }} \mathrm{d} s .
\end{aligned}
$$

${ }^{2}$ As shown in Tagger et al. (1990) the fast magnetosonic wave does not propagate in the corotation region.
Equation (17) is equivalent to the variational form derived in TP99, but the vertical integration (rather than the approximation of an infinitely thin disk) will give us access to the emission of Alfvén waves. The first five terms of Eq. (17) appear to be obviously hermitian, although they hide the imaginary contribution from the corotation resonance (i.e. the growth or damping term corresponding to the energy exchanged with the Rossby vortex) which will be discussed in the next subsection.

The right-hand side is formed of boundary terms, obtained in the integrations by parts. All these terms are easily shown to be imaginary (i.e. correspond to growth or damping), if the boundaries are far enough that the radial and vertical derivatives can be estimated in a WKB approximation, $\partial_{s}=i k_{s}, \partial_{z}=$ $i k_{z}$, and if waves do propagate at the boundaries, i.e. if the wavevectors are real so that waves can effectively transport energy away.

The first bracket corresponds to the flux of the wave at the radial boundaries; as explained in TP99, a wide range of boundary conditions at the inner disk edge allow the waves to be reflected without loss of energy, i.e. this term vanishes or remains real at $s_{\min }$. At $s_{\max }$ it gives the flux of an outgoing wave, responsible for the usual Swing amplification of spiral waves (driven by self-gravity in galaxies, by pressure in the Papaloizou-Pringle instability (Papaloizou \& Pringle 1985), or by magnetic stresses in Tagger et al. 1990).

The last term is new and corresponds to a flux at the vertical boundaries, i.e. the flux of the Alfvén waves emitted vertically. This is confirmed by the fact that this term is associated with the torsional $(\Psi)$ component of the perturbations.

\subsection{Corotation resonance}

In our variational form, Eq. (17), the corotation resonance does not appear explicitly as it did in the equivalent form of TP99: we do not get denominators containing $\tilde{\omega}$, vanishing at corotation. But the corotation resonance must of course be present, since the physics described here is more general than in TP99: it is hidden here in the singularity of $\Psi$ : Eq. (15) has a singular point at corotation (where $\tilde{\omega}$ vanishes) because the highestorder radial derivative of $\Psi$ is proportional to $\tilde{\omega}^{2}$ (assuming, as will be checked a posteriori, that the vertical derivative vanishes at corotation), while the terms in $\Phi$ are proportional to $\tilde{\omega}$. We thus turn, in the vicinity of corotation, to a Frobénius expansion of the form:

$\Psi=a_{-1} \tilde{\omega}^{-1}+a_{n} \tilde{\omega}^{n}+b_{-1} \tilde{\omega}^{-1} \ln \tilde{\omega}+b_{n} \tilde{\omega}^{n} \ln \tilde{\omega}$
$\Phi=c_{0}+c_{n} \tilde{\omega}^{n}+d_{1} \tilde{\omega} \ln \tilde{\omega}+d_{n} \tilde{\omega}^{n} \ln \tilde{\omega}$

where the coefficients $a, b, c, d$ depend on $z$. We first note that, in Eqs. (14), (15), the force terms (in the right-hand sides) are small in the disk, since they are of order $k^{2} V_{\mathrm{A}}^{2} / \Omega^{2}$ (where $k$ is a radial or vertical scale length) compared to the other ones. Since we consider that $\beta=2 c_{\mathrm{S}}^{2} / V_{\mathrm{A}}^{2} \sim 1$, and $c_{\mathrm{s}} \sim h \Omega / r$ where $h$ is the disk thickness, these terms are small near corotation (they play of course their role in the radial propagation of the waves, far from corotation, see TP99). The vertical wavenumbers will be discussed in more details in Sect. 5.1 below. We re-arrange 
Eqs. (14), (15) as:

$$
\begin{aligned}
& \tilde{\nabla}_{\perp}\left(\frac{\tilde{\omega}^{2}-2 \Omega \Omega^{\prime}}{V_{\mathrm{A}}^{2}} \tilde{\nabla}_{\perp} \Phi\right) \\
& +2 m \tilde{\omega} \partial_{s}\left(\frac{\Omega}{V_{\mathrm{A}}^{2}}\right) \Phi-4 m^{2} \frac{\Omega \Omega^{\prime}}{V_{\mathrm{A}}^{2}} \Phi \\
& -2 i \tilde{\nabla}_{\perp}\left(\frac{\Omega}{V_{\mathrm{A}}^{2}} \tilde{\nabla}_{\perp}(\tilde{\omega} \Psi)\right)-i m \partial_{s}\left(\frac{\tilde{\omega}^{2}}{V_{\mathrm{A}}^{2}}\right) \Psi \\
& =-\tilde{\nabla}_{\perp}^{2} \nabla^{2} \Phi
\end{aligned}
$$

and:

$$
\begin{aligned}
& \tilde{\nabla}_{\perp}\left(\frac{\tilde{\omega}^{2}}{V_{\mathrm{A}}^{2}} \tilde{\nabla}_{\perp} \Psi\right)+2 m \tilde{\omega} \partial_{s}\left(\frac{\Omega}{V_{\mathrm{A}}^{2}}\right) \Psi \\
& +2 i \tilde{\omega} \tilde{\nabla}_{\perp}\left(\frac{\Omega}{V_{\mathrm{A}}^{2}} \tilde{\nabla}_{\perp} \Phi\right)+i m \partial_{s}\left(\frac{\tilde{\omega}^{2}}{V_{\mathrm{A}}^{2}}\right) \Phi \\
& =-\partial_{z^{2}} \tilde{\nabla}_{\perp}^{2} \Psi
\end{aligned}
$$

and neglect in the disk the force terms in the right-hand sides. To leading order $\left(\tilde{\omega}^{-1}\right)$ Eq. (20) gives:

$d_{1}=-\frac{i}{\Omega^{\prime}} b_{0}$

while to order $\tilde{\omega}^{0}$ Eq. (21) gives:

$a_{-1} \partial_{s}\left(\frac{W}{V_{\mathrm{A}}^{2}}\right)+m b_{0} \frac{\Omega^{\prime 2}}{V_{\mathrm{A}}^{2}}+2 i m d_{1} \frac{\Omega \Omega^{\prime 2}}{V_{\mathrm{A}}^{2}}=0$

so that

$b_{0}=-\frac{a_{-1}}{m \Omega^{\prime}} \partial_{s} \ln \left(\frac{W}{V_{\mathrm{A}}^{2}}\right)$

and the following coefficients can be derived from the next orders.

The manner in which $\Phi$ and $\Psi$ project on the solutions which are regular and singular at corotation depends on the global solution, which must be obtained numerically as in TP99, of the problem with its boundary conditions. Here we will only use the fact that $\Psi$ has a singular contribution (whose Frobénius expansion starts with $a_{-1} \tilde{\omega}^{-1}$ ) which makes the quadratic form, Eq. (17), non variational at corotation. Combining the singular terms, we find their contribution to the integral:

$$
\begin{aligned}
F_{\text {Corot. }}=\int \mathrm{d} s \int \mathrm{d} z & {\left[\frac{\tilde{\omega}^{2}}{V_{\mathrm{A}}^{2}}\left|\partial_{s} \Psi\right|^{2}\right.} \\
& \left.-2 m \tilde{\omega} \partial_{s}\left(\frac{\Omega}{V_{\mathrm{A}}^{2}}\right)|\Psi|^{2}\right] .
\end{aligned}
$$

Integrating by parts the first term, and retaining only the $\tilde{\omega}^{-1}$ term in the expansion of $\Psi$, we find:

$$
\begin{aligned}
F_{\text {Corot. }} & =-\int \mathrm{d} s \int \mathrm{d} z \frac{\left|a_{-1}\right|^{2}}{\tilde{\omega}^{\star}} \partial_{s}\left(\frac{\rho_{0} W}{B_{0}^{2}}\right) \\
& \approx-\int \mathrm{d} s \frac{\left|a_{-1}\right|^{2}}{\tilde{\omega}^{\star}} \partial_{s}\left(\frac{\Sigma W}{B_{0}^{2}}\right)
\end{aligned}
$$

where $\Sigma$ is the surface density of the disk. As expected we recover the result from TP99 that the resonant contribution to the variational form (and hence to the growth rate of the instability) is proportional to $\partial_{s}\left(\rho W / B_{0}^{2}\right)$, and will give an imaginary contribution from the pole at corotation.

Our goal now is to compute how this singular vorticity generates Alfvén waves, transmitting to the corona a part of the accretion energy and angular momentum extracted from the disk. This flux is readily identified, in Eq. (17), as the last term which represents a contribution from the lower and upper boundaries of our integration domain: this is thus the flux emitted vertically, and we expect that the large amplitude and the singularity of $\Psi$ will give a strong contribution at corotation.

\section{Dispersion relation and Alfvén flux}

\subsection{Dispersion relation in the corona}

The flux of Alfvén waves to the corona appears in our variational form, Eq. (17), as a surface term taken at the lower and upper boundaries of our integration domain:

$F_{\text {Alfven }}=\int_{s_{\min }}^{s_{\max }}\left[\Psi^{\star} \partial_{z} \tilde{\nabla}_{\perp}^{2} \Psi\right]_{-z_{\max }}^{z_{\max }} \mathrm{d} s$.

We take these boundaries far enough above the disk that the density varies smoothly, so that the vertical derivative can be computed in a WKB approximation, $\partial_{z} \approx i k_{z}$. A WKB approximation in the radial direction was also used in TP99 to compute this flux. However, near corotation, the singularity of $\Psi$ makes this radial WKB expansion invalid. We will show this by turning again to a Frobénius expansion, but retaining the force terms in Eqs. (20), (21).

Let us first consider the case of a disk in vacuum: the Alfvén velocity in the corona goes to infinity, so that Eqs. (20), (21) reduce to $\Delta \Phi=0$ and $\partial_{z^{2}} \Psi=0$. In a radial WKB approximation, this gives the result familiar in spiral wave theory, that $\Phi$ varies above the disk as $\exp (-|k z|)$, where $k$ is the horizontal wavenumber, so that $\Phi$ vanishes exponentially. On the other hand one finds that $\Psi$ stays constant with $z$. The full vertical solution, valid across the disk, was given by Tagger et al. (1992). If the coronal density is now small but non vanishing, Eq. (20) shows that far above the disk $\Phi$ must be of the order of $\left(\Omega^{2} /\left(k^{2} V_{\mathrm{A} \infty}^{2}\right) \Psi\right.$, where $k^{-1}$ is the radial scalelength of $\Phi$, and $V_{\mathrm{A} \infty}$ is the Alfvén velocity in the corona, large but not infinite. Thus now $\Phi$ is negligible in Eq. (21), while the vertical derivative of $\Psi$ must be retained. In a WKB approximation in $z$ we now expand this derivative as:

$\partial_{z^{2}} \approx-k_{z}^{2}=-e_{2} \tilde{\omega}^{2}-e_{3} \tilde{\omega}^{3}+\ldots$

and to lowest order of the Frobénius expansion Eq. (21) now gives $e_{2}=0$ and:

$a_{-1} \partial_{s}\left(\frac{W}{V_{\mathrm{A} \infty}^{2}}\right)+m b_{0} \frac{\Omega^{\prime 2}}{V_{\mathrm{A} \infty}^{2}}-2 e_{3} m \Omega^{\prime 2} a_{-1}=0$.

Since the result obtained at the disk, Eq. (24), determines the values of $a_{-1}$ and $b_{0}$ at the base of the corona, we can use them 
to get $e_{3}$ giving finally (assuming for simplicity that the radial profile of $V_{\mathrm{A}}^{2}$ is independent of $z$ ):

$k_{z}^{2} V_{\mathrm{A} \infty}^{2}=\frac{\Omega \tilde{\omega}^{3}}{m \Omega^{\prime 2}} \partial_{s} \ln \left(\frac{W}{V_{\mathrm{A}}^{2}}\right)$.

This result comes as a surprise, for two reasons: the first one is that, since we consider the propagation of a perturbation which is very localized in $s$ (since it is singular), one would have been tempted to use a radial WKB approximation to solve Eq. (21). From the first terms in the left and right-hand sides of this equation, one would get $k_{z}^{2} V_{\mathrm{A} \infty}^{2}=\tilde{\omega}^{2}$, i.e. the familiar dispersion relation of Alfvén waves, Doppler-shifted by differential rotation. The answer here is simple, since with $\Psi \sim \tilde{\omega}^{-1}$ the first term in the right-hand side of Eq. (21) vanishes to lowest order in $\tilde{\omega}^{-1}$. Thus the "usual" dispersion relation applies to regular features, but not to the singular one we are concerned with.

The second surprise is that $k_{z}^{2}$ is proportional to $\tilde{\omega}^{3}$, so that we find $k_{z}$ real only on one side of corotation, depending on the sign of the derivative of $W / V_{\mathrm{A}}^{2}$. Thus the singular perturbation will propagate only on one side of corotation! This can be understood by returning to Eq. (21) and writing it now in terms of $Y=\tilde{\omega} \Psi$, still neglecting the contribution of $\Phi$. We get:

$\tilde{\omega} \tilde{\nabla}_{\perp} \frac{1}{V_{\mathrm{A}}^{2}} \tilde{\nabla}_{\perp} Y+m Y \partial_{s}\left(\frac{W}{V_{\mathrm{A}}^{2}}\right)=k_{z}^{2} \tilde{\nabla}_{\perp}^{2}\left(\frac{Y}{\tilde{\omega}}\right)$.

Let us first neglect the magnetic tension force, on the righthand side. Equation (31) gives, in a radial WKB approximation $\left(\partial_{s} \approx i k\right)$ :

$\tilde{\omega}=\frac{m V_{\mathrm{A}}^{2}}{k^{2}+m^{2}} \partial_{s}\left(\frac{W}{V_{\mathrm{A}}^{2}}\right)$.

This is just the dispersion relation of Rossby waves, Dopplershifted by differential rotation and including the radial gradient of $V_{\mathrm{A}}^{2}$ ! Because this dispersion relation is odd in $\tilde{\omega}$ (whereas more usual ones are even) it allows Rossby waves to propagate only on one side of corotation ${ }^{3}$.

The meaning of Eq. (31) becomes simple: it describes how Rossby waves, forming the singular part of our full solution for $\Psi$, will now propagate upward along the field lines as what we might call Rossby-Alfvén waves. If the radial derivative of $W / V_{\mathrm{A}}^{2}$ is positive (this is the instability criterion of TP99), the wave propagates only beyond corotation and thus carries a positive energy flux, so that its formation in the disk and propagation along the field lines destabilize the negative energy spiral wave inside corotation, which is the main component of our instability.

This makes the physics of the coupling between the instability and Alfvén waves much more complex than the resonance, found by Curry \& Pudritz (1996), between normal modes of the Magneto-Rotational Instability (MRI) and Alfvén waves. The main reason is that they work in a vertical WKB approximation, assuming that the modes have a fixed vertical wavenumber $k_{z}$; it is worth remembering here the result

${ }^{3}$ For a more detailed discussion of Rossby waves with differential rotation, see Tagger (2001). In particular, this shows how a Rossby wave in a disk always collapses to a singularity, the one analyzed here, after a finite time, of the order of a rotation period. of a more complete vertical solution (Tagger et al. 1992): the AEI corresponds to solutions with $n_{z}=0$ nodes across the disk height, whereas the MRI corresponds to solutions with $n_{z} \leq 1$. For $n_{z} \gg 1$, most unstable when $\beta \gg 1$, a vertical WKB approximation can be used. Keeping $k_{z}$ fixed results in finding a resonance away from corotation, at $\tilde{\omega}=k_{z} V_{\mathrm{A}}$, and thus ignoring the coupling with the Rossby vortex. It also ignores the difficulties, encountered here, associated with the vertical variation of the Alfvén velocity.

\subsection{Alfvén waves flux}

We can now compute the flux of Alfvén waves, appearing in the right-hand side of Eq. (17):

$F_{\text {Alfven }}=i \int_{s_{\min }}^{s_{\max }}\left[k_{z}\left|\tilde{\nabla}_{\perp} \Psi\right|^{2}\right]_{z_{\min }}^{z_{\max }} \mathrm{d} s$

where we have assumed that the upper boundary is high enough above the disk, so that the vertical derivative can be replaced by $i k_{z}$. This contribution was computed in TP99, in a radial WKB approximation valid far from corotation. Here our goal is to compute the localized contribution of the resonance, i.e. the flux of Alfvén waves launched from the Rossby vortex, and more precisely the flux associated with the singular part of $\Psi$ as computed in the previous sections. Thus we retain $\Psi \approx a_{-1} \tilde{\omega}^{-1}$ near corotation, and take into account only the region beyond corotation where (if the radial gradient of $W / V_{\mathrm{A}}^{2}$ is positive) the Rossby-Alfvén wave propagates, so that $k_{z}$ is real. We choose for $k_{z}$ the sign of $z$, in order to have waves propagating away at $z_{\min }$ and $z_{\max }$. We get:

$$
\begin{aligned}
F_{\text {Alfven }} \approx 2 i\left|a_{-1}\right|^{2} \quad \int_{0}^{s_{\max }} & \mathrm{d} s \frac{\tilde{\omega}^{3 / 2}}{|\tilde{\omega}|^{4}} \\
& \frac{m^{2}\left|\Omega^{\prime}\right|}{V_{\mathrm{A} \infty}}\left[\Omega \partial_{s} \ln \left(\frac{W}{V_{\mathrm{A}}^{2}}\right)\right]^{1 / 2} .
\end{aligned}
$$

This integral is strongly divergent at corotation, as expected. Various effects can regularize it, in particular the presence of an imaginary part of $\omega$, or the fact that most of our derivations fail at radial scales of the order of the disk half-thickness $h$. Let us consider in particular Eq. (20): we have solved it in the disk by a Frobénius expansion, neglecting altogether the force term, on the right-hand side, although it contains the highestorder radial derivative. Our reason was that this term is small as long as $\tilde{\omega}$ is not too small: remembering that we assume the plasma $\beta$ to be of the order of 1 in the disk, we have:

$V_{\mathrm{A}}^{2} \sim c_{\mathrm{S}}^{2} \sim\left(\frac{h}{r}\right)^{2} \Omega^{2}$

so that this term, acting on the divergent part of $\Phi$, is of the order of

$\left(\frac{h}{r}\right)^{2}\left(\frac{\Omega}{\tilde{\omega}}\right)^{2}$

compared to the one we retain. This means that our expansion breaks down when $\tilde{\omega} \sim(h / r) \Omega$, or $s \sim h / r$, and we will take 
this as the lower bound in our integral, Eq. (34). It is quite possible that the full solution, at $s<h / r$, would still give a divergent result, but then the growth rate of the instability, estimated in TP99 to be

$$
\operatorname{Im}(\omega) \sim\left(\frac{h}{r}\right) \operatorname{Re}(\omega)
$$

would give a similar regularization of the integral. Thus we get a conservative estimate for the flux transported toward the corona by Alfvén waves: assuming that all the gradients are of the order of 1 , we get:

$$
F_{\text {Alfven }} \sim\left|a_{-1}\right|^{2}\left(\frac{r}{h}\right)^{3 / 2} \frac{1}{\Omega V_{\mathrm{A} \infty}} .
$$

On the other hand, the flux deposited at corotation in the vortex (which is the energy removed from the central region of the disk, causing accretion) is given by Eq. (26). As in the classical problem of Landau damping, its imaginary part is given by a Cauchy residue:

$$
F_{\text {Corot. }}=\frac{\left|a_{-1}\right|^{2}}{m\left|\Omega^{\prime}\right|} \partial_{s}\left(\frac{\Sigma W}{B_{0}^{2}}\right) .
$$

Both fluxes are related to the singularity of $\Psi$ at corotation, and thus proportional to $\left|a_{-1}\right|^{2}$. Assuming again for simplicity that $\beta \sim 1$ and that the radial gradients contribute numbers of order unity, we thus get for their ratio a very simple expression:

$$
\frac{F_{\text {Alfven }}}{F_{\text {Corot. }}} \sim\left(\frac{\rho_{\infty}}{\rho_{\mathrm{D}}}\right)^{1 / 2}\left(\frac{r}{h}\right)^{3 / 2}
$$

where we have used $\Sigma \sim \rho_{\mathrm{D}} c_{\mathrm{S}} / \Omega$, and $\rho_{\mathrm{D}}, \rho_{\infty}$ are the densities in the disk and in the corona. The first term in the righthand side is a priori small. It is typical of magnetic braking processes, and leads to a weak efficiency of mechanisms of magnetic origin coupling the disk to the corona. The good surprise for us here is that it is multiplied by the second term, which can be quite large (typically a few hundred to a thousand in the disks of X-ray binaries). The flux ratio can then be a significant fraction of unity, as soon as the corona has a density which is not vanishingly small. For higher density making our estimate larger than 1, a full 3D computation taking into account the finite thickness of the disk would be required.

\subsection{Non-vertical unperturbed magnetic field}

In the body of the paper we have studied the case of a vertical and constant unperturbed field. In Appendix A we discuss the effect of a field which is still straight but depends on the radius. The analytical computation cannot be fully completed, mainly because no simple equilibrium exists without a flow along the field lines; but we show that within reasonable bounds (that the magnetic stress term does not exceed the centrifugal and gravitation forces) our conclusions should not change.

On the other hand a realistic model should also include the curved geometry of the magnetic field, as obtained in jet models. In this case the situation becomes much more complex because in general the three basic MHD waves become coupled by the geometry, in addition to the coupling by differential rotation studied here. In a straight field the Alfvén and slow magnetosonic wave are decoupled: this has allowed us to defer the consideration of slow magnetosonic waves, together with vertical motion, to separate work. In a curved field the Alfvén wave will include vertical motion, and we should in principle include all three components of the displacement and all three MHD waves.

The mixing can still be weak if the scales are very different. For instance, the characteristic wavelength of the slow wave is of the order of $c_{\mathrm{S}} / \tilde{\omega}$, i.e. of the disk scale height if we are not too close to corotation. Thus if the magnetic field is curved on a large scale (of the order of $r$ ), the mixing of the waves is weak and our conclusion should not change much. In particular the Alfvén wave is excited much more efficiently than the slow wave, for which we expect no singular source analogous to the Rossby vortex for the Alfvén wave.

On the other hand more elaborate jet models (e.g. Casse $\&$ Ferreira 2000) show that a slow magnetosonic point forms above the disk. The field lines are sharply bent in its vicinity. But there the Alfven velocity is already much higher than the sound velocity. This disparity should again maintain a separation between slow and Alfvén waves, and allow the latter to propagate the vorticity from the Rossby wave. A realistic computation goes far beyond our present abilities. But we note that the coupling of waves by geometric effects might introduce interesting new channels to deposit energy and momentum from the wave in the corona.

\section{Conclusion}

We have presented a computation of the flux of Alfvén waves emitted to the corona of a magnetized disk by the AccretionEjection Instability. This means that we have justified here the name chosen by TP99: the instability is a spiral density wave, which grows by extracting energy and angular momentum from the disk (thus causing accretion) and transferring them radially outward to the Rossby vortex at corotation; a significant fraction, given by Eq. (38), of this flux is then transmitted vertically to the corona as Alfvén waves. We expect that, if the Alfvén waves can deposit their energy and momentum in the corona, this would be an ideal mechanism to feed a wind or jet directly from the accretion process in the disk.

The amplification of the wave (and thus the flux deposited by the spiral in the vortex) and the flux transmitted to Alfvén waves are both linked to the singularity of the vortex. This allows us to give in a very simple form a result of paramount importance in the physics of accretion disks and jets: an estimate of the fraction of the accretion energy, extracted from the inner region of the disk, which will end up in the corona where it might feed a jet. This fraction is of the order of unity if the coronal density is not too low (typically $10^{-4}$ of the density in the disk would be sufficient, in an X-ray binary).

In order to obtain analytically a physically consistent result, we have had to use a very artificial configuration of the equilibrium magnetic field, vertical and independent of $r$. On the other hand this has allowed us, proceeding rigorously by perturbation of a variational form, to obtain an exact result clarifying the role and the physical nature of the singularity of the 
Rossby vortex at corotation. We can thus expect that these results would survive less stringent assumptions on the equilibrium configuration.

However this must be taken carefully: our final result is in fact divergent at the corotation radius, and regularized by the effect of the finite thickness of the disk, or by the growth rate of the instability. In both cases, it depends on the density in the corona of the disk. Thus we believe that the end result should be obtained from a self-consistent, non-linear description where the growth of the instability itself affects the evolution of the magnetic geometry and the mass loading of the corona.

In this respect it is worth mentioning one of the results of stationary MHD jets models: in these models, as the gas is accelerated along the field lines it passes a slow magnetosonic point where the field lines bend outwards. Magnetocentrifugal acceleration can then proceed and leads to the formation, higher up and further out, of an alfvénic point. The slow magnetosonic point is thus associated with the mass loading of the field lines, and the alfvénic point to the acceleration. By analogy we can thus expect that, while the Alfvén waves described in the present work allow to accelerate the gas, the instability can also generate slow magnetosonic waves which will lift the gas above the disk. The coupling of the instability to the slow wave will be the object of a forthcoming paper.

\section{Appendix A: Radial dependence of $\boldsymbol{B}_{\mathbf{Z}}$}

In the body of the present article we made the simple assumption that the equilibrium field $\boldsymbol{B}^{\mathbf{0}}$ was vertical and constant, allowing us to get an analytical derivation of the Alfven waves flux. In this Appendix we give the general derivation in the case where the field is still straight along $z$ but depends on $s$. We will follow the same computation as in the main text, referring to the corresponding equations.

The first modification appears in the contribution of magnetic stresses $(\boldsymbol{j} \times \boldsymbol{B}) / \rho$. We now have to take into account the equilibrium current, $j^{0}$ :

$f=\frac{\boldsymbol{j}^{0} \times \boldsymbol{B}^{1}}{\rho_{0}}+\frac{\boldsymbol{j}^{1} \times \boldsymbol{B}^{0}}{\rho_{0}}-\left(\boldsymbol{j}^{0} \times \boldsymbol{B}^{0}\right) \frac{\rho_{1}}{\rho_{0}^{2}}$.

We get $\rho_{1}$ from the continuity equation,

$\rho_{1}=-\boldsymbol{\nabla} \cdot\left(\rho_{0} \boldsymbol{\xi}\right)$

which gives after some transformations, and using Eq. (2):

$B_{0} \frac{\rho^{1}}{\rho_{0}}=B_{z}^{1}-\frac{1}{r^{2}} g\left(r B_{0} \xi_{r}\right)$.

Where the function $g$ is defined by:

$g\left(r B_{0} \xi_{r}\right)=\left(\left[\frac{\rho_{0}}{B_{0}}\right]-\frac{B_{0}^{2}}{\tilde{\omega}^{2} \rho_{0}}\left[B_{0}\right] \frac{\partial^{2}}{\partial z^{2}}\right)\left(r B_{0} \xi_{r}\right)$

and $r B_{0} \xi_{r}=-\partial_{s} \Phi+i m \Psi$. Here angular brackets note the logarithmic derivative with respect to $s,[X]=1 / X \partial_{s} X$, and $g$ comes from the departure, due to magnetic pressure, from the keplerian rotation curve. It is weak in the disk for $\beta \sim 1$; it might become important in the corona, but this would require to take into account the velocity flow associated with the formation of the jet. This full computation is beyond our present capabilities.

We thus get the expression of the magnetic stresses:

$$
\begin{aligned}
& B_{0} f_{r}=V_{\mathrm{A}}^{2}\left(\partial_{z} B_{r}^{1}-\partial_{r} B_{z}^{1}\right)-B_{0} \frac{\partial_{r} B_{0}}{\rho_{0}} B_{z}^{1}+B_{0} \frac{\partial_{r} B_{0}}{\rho_{0}} B_{0} \frac{\rho^{1}}{\rho_{0}} \\
& B_{0} f_{\vartheta}=V_{\mathrm{A}}^{2}\left(\partial_{z} B_{\vartheta}^{1}-\partial_{\vartheta} B_{z}^{1}\right) .
\end{aligned}
$$

Expressing this in terms of $\Phi$ and $\Psi$ we get:

$$
\begin{aligned}
& r B_{0}\left[\left(-\tilde{\omega}^{2}+2 \Omega \Omega^{\prime}\right) \xi_{r}+2 i \tilde{\omega} \Omega \xi_{\vartheta}\right]= \\
&-V_{\mathrm{A}}^{2}\left(\partial_{s} \nabla^{2} \Phi-i m \partial_{z^{2}} \Psi\right)-\frac{V_{\mathrm{A}}^{2}}{r^{2}}\left[B_{0}\right] g\left(r B_{0} \xi_{r}\right) \\
& r B_{0}\left[-\tilde{\omega}^{2} \xi_{\vartheta}-2 i \tilde{\omega} \Omega \xi_{r}\right]= \\
&-V_{\mathrm{A}}^{2}\left(\partial_{s, z^{2}} \Psi+i m \nabla^{2} \Phi\right)
\end{aligned}
$$

which generalize Eqs. (10) and (11).

\section{A.1. System}

From these new equations, using the same method as in Sect. 4 we obtain the following parametric system:

$$
\begin{aligned}
& \tilde{\nabla}_{\perp}\left(\lambda \tilde{\omega}^{2} \tilde{\nabla}_{\perp} \Phi\right)-2 \partial_{s}\left(\lambda \Omega \Omega^{\prime} \partial_{s} \Phi\right) \\
& +2 m \partial_{s}(\lambda \Omega \tilde{\omega}) \Phi-2 i \tilde{\nabla}_{\perp}\left(\lambda \tilde{\omega} \Omega \tilde{\nabla}_{\perp} \Psi\right) \\
& -i m \partial_{s}\left(\lambda \tilde{\omega}^{2}\right) \Psi+2 i m \partial_{s}\left(\lambda \Omega \Omega^{\prime} \Psi\right) \\
& =-\lambda V_{\mathrm{A}}^{2} \tilde{\nabla}_{\perp}^{2} \nabla^{2} \Phi \\
& \quad-\left(\lambda \partial_{s} V_{\mathrm{A}}^{2}+V_{\mathrm{A}}^{2} \partial_{s} \lambda\right)\left(i m \partial_{z^{2}} \Psi-\partial_{s} \nabla^{2} \Phi\right) \\
& \quad+\left(\lambda V_{\mathrm{A}}^{2}+\lambda \partial_{s} V_{\mathrm{A}}^{2}+V_{\mathrm{A}}^{2} \partial_{s} \lambda\right) \partial_{s}\left(\frac{\left[B_{0}\right]}{r^{2}} g\left(r B_{0} \xi_{r}\right)\right)
\end{aligned}
$$

and

$$
\begin{aligned}
\tilde{\nabla}_{\perp}\left(\lambda \tilde{\omega}^{2} \tilde{\nabla}_{\perp} \Psi\right)+2 m \tilde{\omega} \partial_{s}(\lambda \Omega) \Psi \\
+2 i \tilde{\nabla}_{\perp}\left(\lambda \tilde{\omega} \Omega \tilde{\nabla}_{\perp} \Phi\right)+i m \partial_{s}\left(\lambda \tilde{\omega}^{2}\right) \Phi \\
+2 i m \lambda \Omega \Omega^{\prime} \partial_{s} \Phi \\
=-\lambda V_{\mathrm{A}}^{2} \partial_{z^{2}} \tilde{\nabla}_{\perp}^{2} \Psi \\
\quad-\left(\lambda \partial_{s} V_{\mathrm{A}}^{2}+V_{\mathrm{A}}^{2} \partial_{s} \lambda\right)\left(i m \partial_{z^{2}} \Psi-\partial_{s} \nabla^{2} \Phi\right) \\
\quad+\left(\lambda V_{\mathrm{A}}^{2}+\lambda \partial_{s} V_{\mathrm{A}}^{2}+V_{\mathrm{A}}^{2} \partial_{s} \lambda\right) \frac{i m}{r^{2}}\left[B_{0}\right] g\left(r B_{0} \xi_{r}\right) .
\end{aligned}
$$

In order to get rid of the term in $\left(i m \partial_{z^{2}} \Psi-\partial_{s} \nabla^{2} \Phi\right)$ we make the same choice as in the constant- $B^{0}$ case, namely $\lambda=V_{\mathrm{A}}^{-2}(s, z)$. The system equivalent to (14), (15) is:

$$
\begin{aligned}
& \tilde{\nabla}_{\perp}\left(\frac{\tilde{\omega}^{2}}{V_{\mathrm{A}}^{2}} \tilde{\nabla}_{\perp} \Phi\right)-2 \partial_{s}\left(\frac{\Omega \Omega^{\prime}}{V_{\mathrm{A}}^{2}} \partial_{s} \Phi\right) \\
& +2 m \partial_{s}\left(\frac{\Omega \tilde{\omega}}{V_{\mathrm{A}}^{2}}\right) \Phi-2 i \tilde{\nabla}_{\perp}\left(\frac{\Omega \tilde{\omega}}{V_{\mathrm{A}}^{2}} \tilde{\nabla}_{\perp} \Psi\right) \\
& -i m \partial_{s}\left(\frac{\tilde{\omega}^{2}}{V_{\mathrm{A}}^{2}}\right) \Psi+2 i m \partial_{s}\left(\frac{\Omega \Omega^{\prime}}{V_{\mathrm{A}}^{2}} \Psi\right) \\
& =-\tilde{\nabla}_{\perp}^{2} \nabla^{2} \Phi+\partial_{s}\left(\frac{\left[B_{0}\right]}{r^{2}} g\left(r B_{0} \xi_{r}\right)\right)
\end{aligned}
$$


and:

$\tilde{\nabla}_{\perp}\left(\frac{\tilde{\omega}^{2}}{V_{\mathrm{A}}^{2}} \tilde{\nabla}_{\perp} \Psi\right)+2 m \tilde{\omega} \partial_{s}\left(\frac{\Omega}{V_{\mathrm{A}}^{2}}\right) \Psi$

$+2 i \tilde{\nabla}_{\perp}\left(\frac{\tilde{\omega} \Omega}{V_{\mathrm{A}}^{2}} \tilde{\nabla}_{\perp} \Phi\right)+i m \partial_{s}\left(\frac{\tilde{\omega}^{2}}{V_{\mathrm{A}}^{2}}\right) \Phi$

$+2 i m \frac{\Omega \Omega^{\prime}}{V_{\mathrm{A}}^{2}} \partial_{s} \Phi$

$=-\partial_{z^{2}} \tilde{\nabla}_{\perp}^{2} \Psi+\frac{i m\left[B_{0}\right]}{r^{2}} g\left(r B_{0} \xi_{r}\right)$.

\section{A.2. The variational form}

After some algebra, integrating by parts and grouping terms we get the equivalent of Eq. (17)

$$
\begin{aligned}
& \int_{s_{\min }}^{s_{\max }} \int_{z_{\min }}^{z_{\max }}\left\{\frac{\tilde{\omega}^{2}}{V_{\mathrm{A}}^{2}}\left(\left|\tilde{\nabla}_{\perp} \Psi\right|^{2}-\left|\tilde{\nabla}_{\perp} \Phi\right|^{2}\right)\right. \\
& +2 \frac{\Omega \Omega^{\prime}}{V_{\mathrm{A}}^{2}}\left|\partial_{s} \Phi\right|^{2} \\
& +2 m \partial_{s}\left(\frac{\tilde{\omega} \Omega}{V_{\mathrm{A}}^{2}}\right)|\Phi|^{2} \\
& -2 m \tilde{\omega} \partial_{s}\left(\frac{\Omega}{V_{\mathrm{A}}^{2}}\right)|\Psi|^{2} \\
& -\Phi^{\star} \tilde{\nabla}_{\perp}^{2} \nabla^{2} \Phi \\
& +\Phi^{\star} \partial_{s}\left(\frac{\left[B_{0}\right]}{r^{2}} g\left(r B_{0} \xi_{r}\right)\right) \\
& \left.-i m \Psi \frac{\left[B_{0}\right]}{r^{2}} g\left(r B_{0} \xi_{r}^{\star}\right)\right\} \mathrm{d} s \mathrm{~d} z \\
& =-\left[\int _ { z _ { \operatorname { m i n } } } ^ { z _ { \operatorname { m a x } } } \frac { \mathrm { d } z } { V _ { \mathrm { A } } ^ { 2 } } \left(\tilde{\omega}^{2} \Phi^{\star} \tilde{\nabla}_{\perp} \Phi-2 \Omega \Omega^{\prime} \Phi^{\star} \partial_{s} \Phi\right.\right. \\
& -\tilde{\omega}^{2} \Psi \tilde{\nabla}_{\perp} \Psi^{\star}+2 i m \Omega \Omega^{\prime} \Phi^{\star} \Psi \\
& \left.\left.-2 i \tilde{\omega} \Omega\left(\Psi \tilde{\nabla}_{\perp} \Phi^{\star}+\Phi^{\star} \tilde{\nabla}_{\perp} \Psi\right)\right)\right]_{s_{\min }}^{s_{\max }} \\
& +\int_{s_{\min }}^{s_{\max }}\left[\Psi^{\star} \partial_{z} \tilde{\nabla}_{\perp}^{2} \Psi\right]_{-z_{\max }}^{z_{\max }} \mathrm{d} s .
\end{aligned}
$$

The only new terms compared to Eq. (17) are the ones containing the function $g$. One easily checks, by expanding $g$ and $\xi_{r}$, that these terms are also hermitian. Therefore A.11 is also a variational form. We can draw the same conclusion as in the constant- $B^{0}$ case.

\section{A.3. Dispersion relation and Alfvén flux}

Taking into account the gradient of $B^{0}$ makes the analytical derivation of the dispersion relation and Alfvén flux much more complex and we will not attempt it here.

If we make the additional assumption that $B_{0}$ depends on $r$ only weakly, i.e. that the current $j^{0}$ is weak, we can get rid of the influence of the terms containing $g$. This assumption is equivalent to requiring that the magnetic stress term is at most of the order of the centrifugal force, which should be the case in a realistic jet model including an equilibrium flow along the field lines. Using $V_{\mathrm{A} \infty}$ as the Alfvén velocity in the corona we obtain the condition:

$\eta(s)=\left[B_{0}\right] V_{\mathrm{A} \infty}^{2} \sim r^{2} \Omega^{2}$.

In this manner the conclusion presented in the body of this article for a constant vertical $B^{0}$ field can be extended to a weakly varying $B_{z}^{0}$.

\section{References}

Blandford, R. D., \& Payne, D. G. 1982, MNRAS, 199, 883

Casse, F., \& Ferreira, J. 2000, A\&A, 353, 1115

Caunt, S., \& Tagger, M. 2001, A\&A, 376, 1095

Curry, C., \& Pudritz, R. E. 1996, MNRAS, 281, 119

Ferreira, J., \& Pelletier, G. 1993a, A\&A, 276, 625

Ferreira, J., \& Pelletier, G. 1993b, A\&A, 276, 637

Ferreira, J., \& Pelletier, G. 1995, A\&A, 295, 807

Fridman, A. M., Khoruzhii, O. V., Lyakhovich, V. V., et al. 2001, A\&A, 371, 538

Lovelace, R. V. E., Wang, J. C. L., \& Sulkanen, M. E. 1987, ApJ, 315, 504

Papaloizou, J. C. B., \& Pringle, J. E. 1985, MNRAS, 213, 799

Pelletier, G., \& Pudritz, R. E. 1992, ApJ, 394, 117

Rodriguez, J., Varnière, P., Tagger. M., \& Durouchoux, P. 2002, A\&A, 387,487

Tagger, M., Henriksen, R. N., Sygnet, J. F., \& Pellat, R. 1990, ApJ, 353,654

Tagger, M., Pellat, R., \& Coroniti, F. C. 1992, ApJ, 393, 708

Tagger, M., Pellat, R., \& Coroniti, F. 1992, ApJ, 393, 708

Tagger, M., \& Pellat, R. 1999, A\&A, 349, 1003 (TP99)

Tagger, M. 2001, A\&A, 380, 750

Varnière, P., Rodriguez, J., \& Tagger, M. 2002, A\&A, 387, 497 